\title{
A FILTERING APPROACH TO UNDERDETERMINED BLIND SOURCE SEPARATION WITH APPLICATION TO TEMPOROMANDIBULAR DISORDERS
}

\author{
Clive Cheong Took, Saeid Sanei, and Jonathon Chambers \\ Centre of Digital Signal Processing, Cardiff University, Cardiff CF24 3AA, Wales, UK, \\ Phone: +44-29-20879063, Email: \{cheongc, saneis, chambersj \}@cf.ac.uk.
}

\begin{abstract}
This paper addresses the underdetermined blind source separation problem, using a filtering approach. We have developed an extension of the FastICA algorithm which exploits the disparity in the kurtoses of the underlying sources to estimate the mixing matrix and thereafter the recovery of the sources is achieved by employing the $\ell 1$-norm algorithm. Also, we demonstrate how promising FastICA can be to extract the sources, without utilizing the $\ell 1$-norm algorithm. Furthermore, we illustrate how this scenario is particularly suitable to the separation of the temporomandibular joint (TMJ) sounds, crucial in the diagnosis of temporomandibular disorders (TMDs).
\end{abstract}

\section{INTRODUCTION}

Blind source separation (BSS) is the problem of estimating the source signals from their mixtures, without explicit a priori knowledge of the medium and the source signals. If the number of mixtures is less than the number of sources, the problem is termed as underdetermined BSS (UBSS). Typically, it comprises of two stages: 1) blind identification of the mixing matrix 2) source extraction. Mathematically, BSS can be defined on the basis of the following generative model:

$$
\mathbf{x}(t)=\mathbf{A s}(t)+\mathbf{v}(t)
$$

where $\mathbf{x}(t)$ represents the mixture signals detected by $m$ sensors at discrete time instant $t$, i.e. $\mathbf{x}(t)=\left[x_{1}(t), x_{2}(t), \ldots\right.$ , $\left.x_{m}(t)\right]^{T} \in \Re^{m}$, and the source vector $\mathbf{s}(t)=\left[s_{1}(t), s_{2}(t), \ldots\right.$ , $\left.s_{n}(t)\right]^{T} \in \Re^{n}$ where $T$ denotes the transpose operation. A is the instantaneous mixing matrix (of size $m \times n$ ) that reflects the mixing characteristics of the medium, while $\mathbf{v}(t)$ represents the additive noise vector. However, we do not consider $\mathbf{v}(t)$ because the noise is modelled as an additional source. We employ both sparse and independent component analysis (SCA and ICA). ICA is a tool for BSS with the assumption that the sources are independent and unmixing is

Thanks to Dental Institute of King's College London for providing the TMJ signals achieved by $\mathbf{y}(t)=\mathbf{B} \mathbf{x}(t)$, where $\mathbf{B}$ is the so-called separating matrix (of size $n \times m$ ) and $\mathbf{y}(t)$ is a vector of dimension $n$ containing the independent components at discrete time instant $t$. Likewise, SCA estimates the sparsest solution $\mathbf{s}(t)$ pertaining to (1) [1]. We address the problem of underdetermined BSS, where $\mathbf{A}$ is a $2 \times 3$ matrix as this pertains to the problem of separating temporomandibular joint sounds from two noisy observations (two sensor/mixture signals and three source signals). As in many standard ICA and SCA algorithms, we make the following assumptions: A1: All the source signals are mutually statistically independent and super-Gaussian.

A2: The mixing matrix is full column rank.

A3: $\operatorname{kurt}\left(s_{2}\right), \operatorname{kurt}\left(s_{3}\right) \gg \operatorname{kurt}\left(s_{1}\right)$ where $s_{i}$ is the $i$ th source and $k u r t($.$) denotes the kurtosis.$

A4: The two highly super-Gaussian source signals are sparse. A5: The third source signal (with low kurtosis) has a substantially lower variance as compared to the other two sources, i.e. $\operatorname{var}\left(s_{2}\right), \operatorname{var}\left(s_{3}\right) \gg \operatorname{var}\left(s_{1}\right)$ where $\operatorname{var}($.$) denotes$ the variance.

The organization of the paper is as follows; the next section deals with the background of the TMD BSS problem. Section 3 provides the details of our algorithm with justifications of its steps. Section 4 evaluates the performance of our algorithm against two algorithms, notably k-means clustering [2] and the algorithm of Li et al. [1]. Lastly, we demonstrate that FastICA can be promising to extract such sources, followed by our conclusions in Section 5 .

\section{BACKGROUND}

\subsection{Sparsity}

Sparsity refers to the situation where a relatively small number of source signals is active over any particular time interval. For the case of a single active source, sparsity can be mathematically described as:

$$
\begin{gathered}
\left\{s_{i}(t) ; i=1, \ldots, n\right\} \\
s_{i}(t) \neq 0 \text { when } s_{i}(t)>>s_{j}(t) \forall j \neq i
\end{gathered}
$$


where $s_{i}$ is a given source signal and $s_{j}$ is another source signal.

\subsection{FastICA}

This algorithm has not previously been employed to solve the underdetermined BSS in the context of TMD. FastICA maximizes the non-Gaussianity of the signals by utilizing higher order statistics such as kurtosis and negentropy (Neg(.)). That will enable the FastICA to determine a vector $\mathbf{b}=$ $\left[b_{1}, b_{2}, \ldots, b_{m}\right]^{T} \in \Re^{m}$. Note that negentropy can be viewed as a measure of deviation from a Gaussian distribution. Consider the case where there are two mixture signals $\mathbf{x}(\mathrm{t})=$ $\left[x_{1}(t) ; x_{2}(t)\right]^{T} . \mathbf{b}_{i}$ is the best possible linear combination of the mixture signals $x_{1}(t)$ and $x_{2}(t)$ to yield an independent component $y_{1}(t)$ with the maximum kurtosis.

$$
y_{1}(t)=\arg \sup _{b_{1}, b_{2}}\left(N e g\left(b_{1} x_{1}(t)+b_{2} x_{2}(t)\right)\right)
$$

FastICA searches for another $\mathbf{b}_{i i}$ which is orthogonal to the one previously found by utilizing deflationary orthogonalization.

$$
\mathbf{b}_{i i} \longleftarrow \mathbf{b}_{i i}-\frac{\mathbf{b}_{i}^{T} \mathbf{b}_{i i}}{\mathbf{b}_{i}^{T} \mathbf{b}_{i}} \mathbf{b}_{i}
$$

Whenever these independent components fail to be the source signals, FastICA has in fact achieved projection pursuit [3]. In analogy to least squares schemes which focus on high variance signals, when the FastICA algorithm is applied to UBSS mixtures of sources with highly disparate kurtoses (assumption A3), the performance of the ICA algorithm will be centered on those sources with maximum kurtoses.

\subsection{The Temporomandibular Disorder problem}

An illustration of a $2 \times 3$ UBSS scenario portrays itself in the source separation of TMJ sounds. TMD is a collective term referring to medical problems related to the region of the mandible (lower jaw) and the temporal bone (skull). Click and crepitus are the two well-known TMJ sounds. The click is normally associated with the perforation of the disc between the mandible and the temporal bone. Also, the crepitus hints the presence of a degenerative joint disease (e.g. osteoarthrosis). Poor detection of these sounds leads to misdiagnosis of TMDs. The dental specialist has to distinguish between the TMJ sounds such as click, crepitus and the background noise/interferences such as movement of the muscle (e.g. tongue). But the classification of these TMJ sounds is inherently subjective and hard for the clinicians to determine the correct pathology. This has led to controversy as in $[4,5]$. Thus, it is particularly difficult for the dental specialist to diagnose TMD, when click, crepitus and background noise are all present within the TMJ sounds. For more information on TMDs, the reader should refer to [5].

\section{DEVELOPMENT OF THE ALGORITHM}

In this section, we outline the underdetermined BSS algorithm, and justify each step. Recall that our BSS problem is constrained to 3 source signals and 2 mixture signals, with the assumptions made in section $1 . M$ denotes the window size of the filters in terms of samples.

1. Temporally median filter (5) the mixture signals $\mathbf{X}$ to yield $\mathbf{F}$. The objective is to attenuate the effects of the outliers (out of range peaks) with a small window of 3 samples. This results in a better estimation of $\mathbf{A}$ because FastICA relies on higher order statistics (kurtosis and negentropy), which in turn are sensitive to outliers. The importance of attenuating these outliers has been illustrated in [6].

$$
y[t]=\operatorname{median}_{k=-(M-1) / 2}^{k=}\{x[t+k]\}
$$

2. Apply FastICA to $\mathbf{F}$ to estimate two columns of the mixing matrix A. Due to $\operatorname{kurt}\left(s_{2}\right), \operatorname{kurt}\left(s_{3}\right) \gg \operatorname{kurt}\left(s_{1}\right)$, the two highly super-Gaussian sources are estimated and thereby their corresponding columns in A are estimated. The key observation is that in UBSS FastICA will focus on the high kurtoses sources.

3. Temporally mean filter (6) $\mathbf{F}$ with $M$ greater than twice the duration of the maximum period $P_{\max } \mathrm{du}-$ ring which the two highly super-Gaussian sources are active. Although $M$ is not known a priori, a large window can be used due to assumption A4. Mean filtering will mitigate the presence of the two highly super-Gaussian source signals in both mixtures.

$$
g[t]=\frac{1}{M} \sum_{k=t-M+1}^{t} h[k]
$$

Consider the periods during which the two highly superGaussian source signals are active. Over these periods, we note the predominance of these two source signals in the mixture signals. Similarly, when these highly super-Gaussian sources are not active the third source predominates in the mixture signals. Hence, average-filtering with $M \geq 2 P_{\max }$ increases the weighting of the weakly super-Gaussian source signal in the mixture signals during these periods over which the highly super-Gaussian source signals are active.

4. Apply FastICA to estimate the third column of A pertaining to the weakly super-Gaussian source signal. Owing to the suppression of the two highly superGaussian sources in the mixtures by mean filtering, 
FastICA will compute the estimation of the last column of A. Due to its equivalence to projection pursuit (PP) [3], FastICA may fail to estimate the column of A corresponding to the weakly super-Gaussian source. PP can be modelled as:

$$
\mathbf{Z}=\mathbf{K X}
$$

where $\mathbf{Z}$ is the projected data, and $\mathbf{K}$ is the linear transformation of the data $\mathbf{X}$. PP is an exploratory data technique projecting our raw data into a space where the feature of interest (i.e. non-Gaussianity) is maximized.

Thus, we estimate two independent components instead of one. Thereafter, the column corresponding to the independent component with the minimum kurtosis is selected (due to the assumption A5) to form the last column of the estimate of $\mathbf{A}$, i.e. $\hat{\mathbf{A}}$.

5. Apply the $\ell 1$-norm algorithm [1] to extract the source signals.

6. If the $\ell 1$-norm algorithm fails to yield satisfactory estimates of the sources, remove the mean-filtering (8) of the last independent component found, i.e. the estimate of the least super-Gaussian source. Although, we might expect this component to be a linear mixture of the three sources, the source with the minimum kurtosis is the predominant constituent (owing to the suppression of the two highly super-Gaussian sources by mean-filtering).

$$
p[t]=M q[t]-\sum_{k=t-M+1}^{t-1} p[k]
$$

Therefore, to strengthen the presence of the source with the maximum kurtosis as the independent component, we can substract or add any two distinct scaled version of the source with the maximum kurtosis. Applying FastICA to the two new mixtures improves the estimate of the weakly super-Gaussian source. This step is required, as the estimate of the super-Gaussian noise of step 4 comprises of a significant influence of the most super-Gaussian source, but not high enough for FastICA to separate it from the super-Gaussian noise. See the first two plots of Figure 3. Also, we already have estimates of the highly non-Gaussian sources from step 2 .

\section{EXPERIMENTAL RESULTS}

In our study, we have considered the TMJ sounds to be mixtures of the click, crepitus and a super-Gaussian noise.
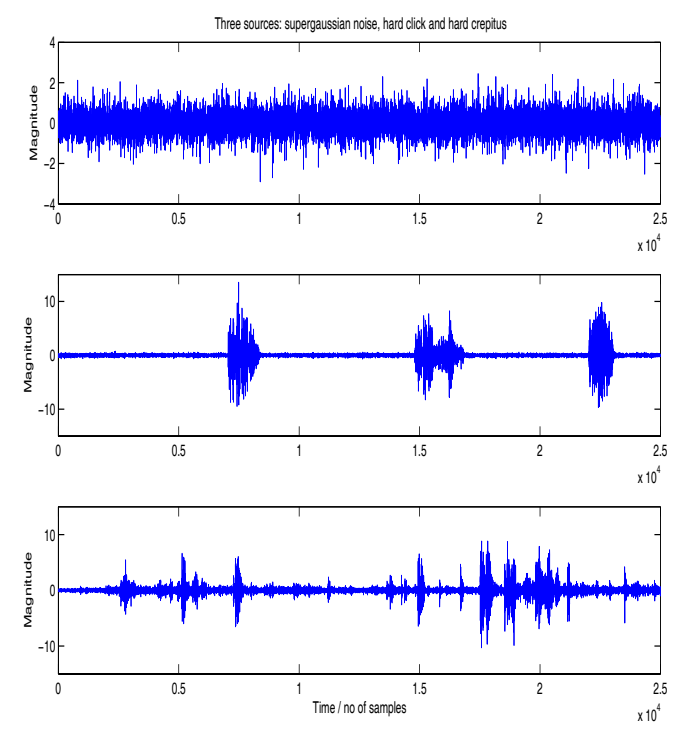

Fig. 1. The super-Gaussian noise, the click and the crepitus. Note the sparsity of the click (middle plot) where the click occurs in the three excitation regions. Likewise for the crepitus signal (last plot).

Thus, the task is to separate the click, the crepitus and the super-Gaussian noise present within the two TMJ sound mixtures. The click and crepitus were recorded using a special stethoscope connected to a microphone placed at the openings of the auditory canals to record the TMJ sounds sampled at $8000 \mathrm{~Hz}$. To model the noise, we generated one with Laplacian distribution described by the following equation:

$$
P(s)=\frac{1}{2 \lambda} \exp \left\{-\frac{|s-\theta|}{\lambda}\right\}
$$

where the variance $\sigma^{2}=2 \lambda^{2}$ and the mean $\mu=\theta$.The performance measure $(P M)[6]$ is employed here to provide an indication of the difference between the actual mixing matrix $\mathbf{A}$ and the estimated $\hat{\mathbf{A}}$. However, $P M$ requires both $\mathbf{A}$ and $\hat{\mathbf{A}}$ to have unit norm columns. $0 \leq P M \leq 1$. $P M$ equals to 0 if $\hat{\mathbf{A}}=\mathbf{A P}$ where $\mathbf{P}$ is a permutation matrix.

$P M(\mathbf{A}, \hat{\mathbf{A}})=1-\left(\frac{1}{2 n} \sum_{i=1}^{n} \sup _{j}\left|\mathbf{A}^{T} \hat{\mathbf{A}}\right|_{i j}+\frac{1}{2 n} \sum_{j=1}^{n} \sup _{i}\left|\mathbf{A}^{T} \hat{\mathbf{A}}\right|_{i j}\right)$

The kurtoses of the noise, click, and crepitus (measured when each source exists separately) are respectively 3.0, 23.7 and 14.4. Figure 1 shows the sources, i.e. the weakly super-Gaussian noise, click and crepitus from top to bottom. A (generated randomly from a normal distribution) is given 
below:

$$
\left(\begin{array}{ccc}
0.6233 & -0.7900 & 0.9999 \\
0.7820 & -0.6131 & -0.0139
\end{array}\right)
$$

The performance of our extended algorithm of FastICA was measured against the k-means clustering [2] and the algorithm of Li et al. [1] as the SNR was varied (shown in Figure 2). Thereafter, we visually demonstrate the potential of step 6 of the extended FastICA algorithm to extract the sources in Figure 3.

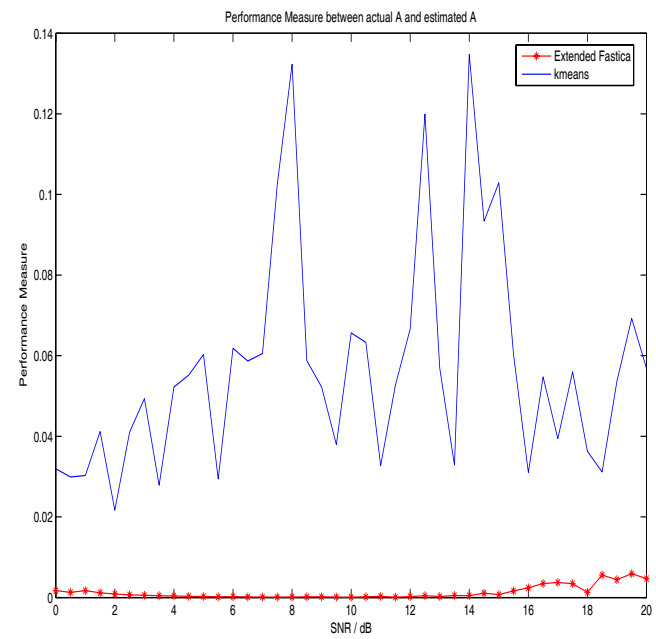

Fig. 2. $P M$ of our extended FastICA plotted in '-*' and that of the k-means clustering in '-' against SNR in $\mathrm{dB}$. The algorithm of $\mathrm{Li}$ failed to estimate the mixing matrix $\mathrm{A}$

Remarks : The k-means algorithm has a reasonable performance when its maximum $P M$ is less than 0.14 . The fluctuation of the performance of the k-means algorithm arises due to the initialization of the algorithm on which its convergence depends. Our algorithm outperforms k-means (indicated by its much lower performance measure). However, the algorithm of $\mathrm{Li}$ [1] failed to estimate the mixing matrix A. This is probably because the super-Gaussian noise source is permanently active. One of the requirement of Li's algorithm is sparsity with respect to all the three signals.

\section{CONCLUSIONS}

This study has shown how filtering can assist in solving the underdetermined blind source separation in the context of TMDs. Although the common approach is geometrical such as the k-means and the algorithm of $\mathrm{Li}$ [1] that exploit sparsity, our algorithm takes advantage of both the sparsity and the statistical entities of the source signals. In that respect, our algorithm is more efficient in its solutions than the kmeans and Li's algorithms. Besides, the superiority of our algorithm with respect to the k-means and Li's algorithm has been shown. We have also illustrated that we can exploit the ability of FastICA in solving such an UBSS problem.
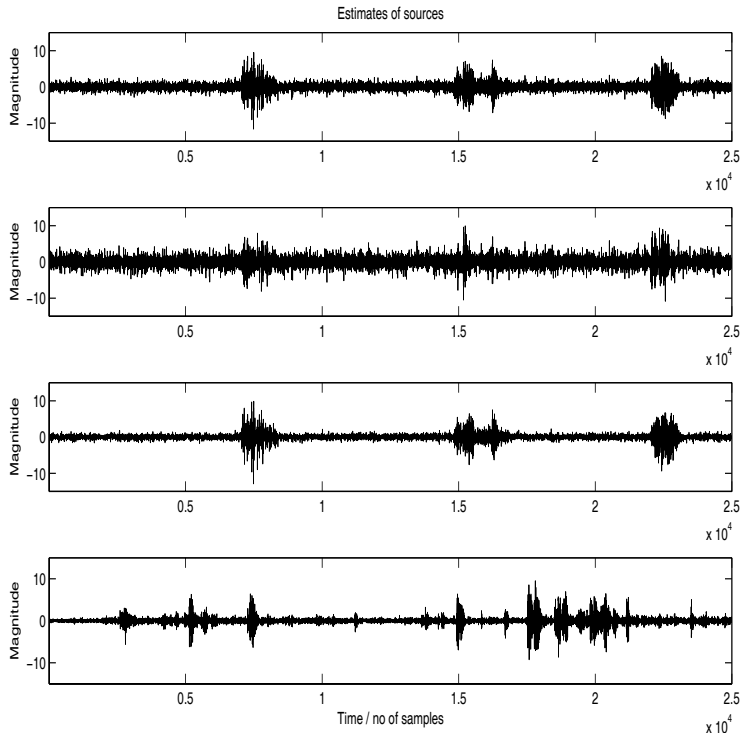

Fig. 3. From top to bottom: Estimate of noise of step 4, final estimate of noise, click and crepitus. Note the presence of the most super-Gaussian source click in the first estimate of the noise.

\section{REFERENCES}

[1] Y. Li, S. Amari, A. Cichocki, and D. W. .C. Ho, "Underdetermined Blind Source Separation based on Sparse Representation," IEEE Transactions in Signal Processing, vol. 54, no. 2, Feb 2006.

[2] S. Roberts and R. Everson, Independent Component Analysis, Cambridge University Press, 2001.

[3] A. Hyvarinen, J. Karhunen, and E. Oja, Independent Component Analysis, John Wiley \& Sons, INC, 2001.

[4] C. S. Greene, N. D. Mcneill, C. Clark, and G. T. Truelove, "Temporomandibular disorders and science: a response to the critics," Journal of Prosthetic Dentistry, vol. 80, pp. 214, 1998.

[5] R. J .M. Gray, S. J.Davies, and A. A. Quayle, Temporomandibular Disorders: A Clinical Approach, 1st Ed., British Dental Association, 1995.

[6] F. C. Meinecke, S. Harmeling, and K. R. Muller, "Robust ICA for Super-Gaussian Sources," Independent Component Analysis and Blind Source Separation: Fifth International Conference, vol. 5, pp. 217-224, 2004. 